\title{
THE BOUNDEDNESS OF CERTAIN SETS OF ORTHO- NORMAL POLYNOMIALS IN ONE, TWO, AND THREE VARIABLES
}

\author{
BY \\ DUNHAM JACKSON
}

1. Introduction. When a system of orthonormal polynomials has been constructed on an algebraic curve, convergence of the development of an "arbitrary" function in terms of these polynomials is intimately associated with boundedness of the polynomials themselves as considered in sequence.

On a specified curve, if sets of orthonormal polynomials are constructed with different weight functions the property of boundedness carries over from one weight function to another with a considerable degree of generality. The question whether there is any weight function for which the orthonormal system possesses the property of boundedness appears on the other hand, apart from certain limited principles of generalization, to require separate examination for each type of curve.

The writer has recently discussed from this point of view domains of orthogonality on plane curves of the second degree [7](1). Other loci have been treated by Fulton Koehler [8] in a manner making use of geometrical symmetry of the figure, and still others by Margaret Martin in a thesis, not yet published, at the University of Minnesota.

The present paper is concerned with a succession of rational curves, on each of which the coordinates are given as polynomials, or rather as monomials (not merely as rational functions of more general character) in terms of a parameter. (In the cases first considered the parameter is itself merely one of the coordinates.) The desired conclusions are obtained in each instance through study of an appropriate system of orthogonal polynomials in the parameter. While the orthogonal systems in a single variable thus brought up for consideration may in themselves have the appearance of a somewhat haphazard collection of isolated examples, they are brought together as aspects of an inclusive problem through their interpretation as polynomials associated with a curve in two or three dimensions. Even so it must be admitted that the selection of topics is guided by opportunist principles, and anything in the nature of a final comprehensive theory is still far from attainment. Although certain partial generalizations are not far to seek, they are still of such limited scope in proportion to the amount of labor involved in a formal presentation that preference has been given to the comparative simplicity and vididness of specific illustrations.

Presented to the Society, February 24, 1945; received by the editors January 1, 1943.

(1) Numbers in brackets refer to the bibliographical citations at the end of the paper. 
2. The curves $y=x^{3}, y=x^{m}$; permutation of powers of $x$. In connection with the curve $y=x^{2}$ it has been pointed out $[7$, p. 361] that the independent monomials for the application of the Schmidt process may be taken as

$$
1, x, y, x y, y^{2}, x y^{2}, y^{3}, \cdots \text {. }
$$

These reduce on the curve to the successive powers of $x$, and the resulting orthogonal polynomials for an arc of the parabola are merely Legendre polynomials for the corresponding interval of values of $x$.

For the curve $y=x^{3}$, since any term containing the third or a higher power of $x$ can be replaced by one of lower degree by means of the equation of the curve, the independent monomials may be taken as

$$
1, x, y, x^{2}, x y, y^{2}, x^{2} y, x y^{2}, y^{3}, x^{2} y^{2}, x y^{3}, y^{4}, \cdots .
$$

In terms of $x$ these are

$$
1, x, x^{3}, x^{2}, x^{4}, x^{6}, x^{5}, x^{7}, x^{9}, x^{8}, x^{10}, x^{12}, \cdots ;
$$

they constitute again the sequence of non-negative integral powers of $x$, without omission, but with the exponents permuted now from their natural order. The question presents itself then whether operation of the Schmidt process on the powers of $x$ in the order indicated produces a bounded system of or thonormal functions. A permutation having the effect merely of interchanging monomials which are of like degree in $x$ and $y$ together would be a matter of no concern for the theory of the orthogonal system on the curve; in fact the set of orthonormal polynomials of any specified degree in the pair of variables is not significantly distinguishable from any other set obtained from it by an orthogonal substitution; see [2, pp. 233-234]. But unrestricted permutation of the basic functions to which the Schmidt process is applied gives rise in general to wholly different orthogonal systems and different developments in series.

The answer is immediate, to be sure, for a domain of integration symmetric with respect to the origin, $-a \leqq x \leqq a$, with $x$ as variable of integration and with unit weight function. For the even exponents taken by themselves are in natural order, and the same is true of the odd exponents; the Schmidt process applied to the even powers by themselves or to the odd powers by themselves gives the normalized Legendre polynomials of even and odd degrees respectively for the interval $(-a, a)$, uniformly bounded except near the ends of the interval; and since any even polynomial is orthogonal to any odd polynomial, the complete orthonormal system is obtained by taking the two sets together. If $p_{n}(x)$ is the normalized polynomial of the $n$th degree for the interval $(-a, a)$, the orthonormal system formed from the sequence (1) consists in order of the polynomials

$$
p_{0}(x), p_{1}(x), p_{3}(x), p_{2}(x), p_{4}(x), p_{6}(x), p_{5}(x), p_{7}(x), p_{9}(x), \cdots .
$$


Consider now an arbitrary interval $a \leqq x \leqq b$. It is no longer possible to proceed by dealing with even and odd powers separately. Let $p_{n}(x)$ be the normalized Legendre polynomial of the $n$th degree (normalized polynomial of the orthogonal system with unit weight function) for the interval $(a, b)$, and let $q_{0}(x), q_{1}(x), q_{2}(x), \cdots$ be the successive members of the orthonormal system formed from the sequence (1). It is obvious (in the sense of being immediately demonstrable) that $q_{n}(x)$ is a polynomial of degree $n+1$ at most. Hence $q_{n}(x)$ can be expressed as a linear combination of $p_{n+1}(x), p_{n}(x), \cdots$, $p_{0}(x)$. Conversely, each power $x^{k}$ appears in the leading term of a polynomial $q_{n}(x)$ with index $n$ not greater than $k+1$ (the leading term, that is to say, according to the arrangement in the sequence (1)) and is linearly expressible in terms of $q_{0}(x), \cdots, q_{k+1}(x)$. By the property of orthogonality of the $q$ 's it is orthogonal to $q_{n}(x)$ if $n>k+1$.

Hence $q_{n}(x)$ is orthogonal to every polynomial of degree less than $n-1$; ir. particular, $q_{n}$ is orthogonal to $p_{0}, p_{1}, \cdots, p_{n-2}$, and is expressible in the form

$$
q_{n}(x)=c_{n, n+1} p_{n+1}(x)+c_{n n} p_{n}(x)+c_{n, n-1} p_{n-1}(x)
$$

with

$$
c_{n k}=\int_{a}^{b} q_{n}(x) p_{k}(x) d x .
$$

By Schwarz's inequality and the normalization of the p's and the $q$ 's,

$$
c_{n k}^{2} \leqq \int_{a}^{b}\left[q_{n}(x)\right]^{2} d x \int_{a}^{b}\left[p_{k}(x)\right]^{2} d x=1 .
$$

From the boundedness of the $p$ 's it follows then that the polynomials $q_{n}(x)$ are uniformly bounded throughout any closed interval interior to $(a, b)$.

It is merely a restatement of the same fact to say that the orthonormal polynomials in $x$ and $y$ on an arc of the curve $y=x^{3}$, with $x$ as variable of integration and with unit weight function, are uniformly bounded except near the ends of the arc. The conclusion is not affected by a change to arc length as variable of integration for the definition of orthogonality, since this is equivalent to changing the weight function by introduction of a factor

$$
d s / d x=\left(1+9 x^{4}\right)^{1 / 2}
$$

which satisfies the conditions for the application of Korous's theorem (see $[9$, p. $157 ; 6$, pp. 205-208]). A minor generalization admits replacement of the equation $y=x^{3}$ by

$$
y=A x^{3}+B x^{2}+C x+D
$$

with arbitrary $A \neq 0, B, C, D$, into which the former can be carried over by the nonsingular affine linear transformation 


$$
x=a x^{\prime}+b, \quad y=c x^{\prime}+y^{\prime}+d,
$$

with $a, b, c, d$ determined successively by the relations

$$
a^{3}=A, \quad 3 a^{2} b=B, \quad 3 a b^{2}-c=C, \quad b^{3}-d=D ;
$$

see $[7$, pp. $353-354]$.

The reasoning of the third and second paragraphs preceding is effective in principle if the sequence (1) is replaced by any permutation of the nonnegative integral powers for which there is a fixed number $r$ such that no power is shifted more than $r$ places from its natural position in the sequence. Under these circumstances the general $q_{n}(x)$ can be expressed in the form

$$
q_{n}(x)=\sum_{k=n-r}^{n+r} c_{n k} p_{k}(x)
$$

with $\left|c_{n k}\right| \leqq 1$, and the $q$ 's are uniformly bounded except near the ends of the interval, in consequence of the corresponding property of the $p$ 's.

The result is applicable at once to the orthonormal polynomials in $x$ and $y$ on an arc of the curve $y=x^{m}$ for arbitrary positive integral $m$. The fact that there is an $r$ for each $m$ is easily recognized; a little more calculation, of the most elementary sort, is involved in obtaining the value $r=(m-1)(m-2) / 2$.

Still more generally, let

$$
\phi_{0}(x), \phi_{1}(x), \phi_{2}(x), \cdots
$$

be an arbitrary sequence of functions of $x$, subject to appropriate conditions of integrability and linear independence, and let $p_{0}(x), p_{1}(x), p_{2}(x), \cdots$ be, the orthonormal system formed from the $\phi$ 's in succession by means of the Schmidt process. Let $q_{0}(x), q_{1}(x), q_{2}(x), \cdots$ be the orthonormal system similarly formed from a permutation of the sequence (2) for which there is a fixed $r$ such that none of the $\phi$ 's is moved more than $r$ places from its original position. By adaptation of the reasoning of the last paragraph but one, each $q_{n}(x)$ is linearly expressible in terms of not more than $2 r+1$ of the $p$ 's, with coefficients not exceeding 1 in absolute value, and boundedness of the $p$ 's on a specified part of the domain of integration implies boundedness of the $q$ 's on the same point set.

3. The curves $y^{2}=x^{3}, y^{2}=x^{5}$, symmetric arc. On the semi-cubical parabola $y^{2}=x^{3}$ the expressions

$$
1, x, y, x^{2}, x y, y^{2}, x^{2} y, x y^{2}, y^{3}, x^{2} y^{2}, x y^{3}, y^{4}, \cdots
$$

may again be used as linearly independent monomials. In terms of a parameter $t$ such that $x=t^{2}, y=t^{3}$, these monomials become

$$
1, t^{2}, t^{3}, t^{4}, t^{5}, t^{6}, \cdots,
$$

the successive powers of $t$ in natural order, with the first power omitted. 
Once more the structure of the orthogonal system is simplified by a special hypothesis of symmetry. For an interval $-a \leqq t \leqq a$, with $t$ as variable of integration and unit weight function, any even polynomial in $t$ is orthogonal to any odd polynomial, and the orthogonal polynomials of even degree and those of odd degree can be calculated separately. The former are the even Legendre polynomials in $t$ for the interval $(-a, a)$. The latter, linear combinations of $t^{3}, t^{5}, \cdots$, are found by constructing the orthogonal polynomials of odd degrees $1,3,5, \cdots$ on the same interval for weight $t^{4}$, and multiplying each of these polynomials by $t^{2}$. When normalized they are uniformly bounded except for $t$ near $-a, 0$, and $a$; see for example [6, pp. 201-203].

The orthogonal polynomials in $x$ and $y$ on the curve are obtained at once by replacing the respective powers of $t$ by the corresponding monomials in terms of the coordinates. The weight function to be sure has been taken as unity for integration with respect to the parameter $t$, as has been usual in the discussion of similar problems, for arc length as variable of integration. While the adjustment to unit weight for integration with respect to $s$ would be easy in this particular instance, it is not the purpose of the present paper henceforth to give systematic attention to questions of change of weight function. A powerful instrument for dealing with this sort of problem, the application of which to orthogonal systems on algebraic curves calls for separate consideration at length, is supplied by a recent paper of Albert and Miller [1].

A similar approach, supplemented by reference to the concluding paragraph of the preceding section, can be used in dealing with a symmetric arc of the curve $y^{2}=x^{5}$. Since any term containing $x^{5}$ as a factor is equal on the curve to one of lower degree, the fundamental sequence of independent monomials can be taken as

$$
\begin{gathered}
1 ; x, y ; x^{2}, x y, y^{2} ; x^{3}, x^{2} y, x y^{2}, y^{3} ; \\
x^{4} y^{j}, x^{3} y^{j+1}, x^{2} y^{j+2}, x y^{j+3}, y^{j+4} ; \quad j=0,1,2, \cdots .
\end{gathered}
$$

In terms of the parametric representation $x=t^{2}, y=t^{5}$, these monomials are powers of $t$ with the successive exponents

$$
\begin{array}{r}
0 ; 2,5 ; 4,7,10 ; 6,9,12,15 ; 8,11,14,17,20 \\
5 j+8,5(j+1)+6,5(j+2)+4,5(j+3)+2,5(j+4) ; \\
\quad j=1,2, \cdots,
\end{array}
$$

so that after the first few entries any two exponents standing 5 steps apart in the sequence differ by 5 units, and if a particular exponent occurs all larger exponents congruent to it modulo 5 are included also. It appears then on further inspection that all the non-negative integral powers of $t$ are represented except the first and third. Furthermore the numbers of exponents preceding $5 j+8,5(j+1)+6, \cdots, 5(j+4)$ in the sequence for $j \geqq 0$ are respectively $5 j+10,5 j+11, \cdots, 5 j+14$, so that the number of places by 
which an exponent is displaced from its natural position in order of magnitude remains finite as the sequence proceeds. Consequently, by the last paragraph of $\$ 2$, the property of boundedness will carry over to the orthonormal polynomials of the present problem if it is established for the orthonormal polynomials constructed from the powers $1, t^{2}, t^{4}, t^{5}, t^{6}, t^{7}, t^{8}, \cdots$ in natural order.

By reason of the symmetry of the interval, defined as before by the inequalities $-a \leqq t \leqq a$, even and odd polynomials can again be considered separately. The orthonormal polynomials of even degree are once more merely the normalized even Legendre polynomials. Those of odd degree are obtained by forming the odd orthonormal polynomials of degrees $1,3,5, \ldots$ for weight $t^{8}$ and multiplying each by $t^{4}$. The polynomials thus constructed are uniformly bounded except near $t=-a, t=0$, and $t=a$. Consequently the same is true of the orthonormal polynomials in $t$ constructed with the sequence of exponents (4); and the polynomials on the curve are obtained by replacing the respective powers of $t$ by the corresponding expressions in terms of $x$ and $y$.

4. The curve $y^{2}=x^{3}$, arbitrary arc. Omission of a power of the independent variable on an arbitrary interval. Let consideration be turned now to an arbitrary segment of the curve $y^{2}=x^{3}$, marked off by the inequalities $a \leqq t \leqq b$. The values of $a$ and $b$ are unrestricted as to sign; the range of integration on the curve may or may not include the cusp as interior or end point. An arbitrary linear combination of the fundamental monomials (3) is an arbitrary polynomial in $t$ with the first power absent, that is, a polynomial $P(t)$ satisfying the auxiliary condition $P^{\prime}(0)=0$. There is a connection therefore with a theory of orthogonal polynomials subject to auxiliary conditions which has already been presented in its preliminary stages $[4,5]$; the existing results are however not adequate for direct application here, and the question of boundedness must be examined in some detail. The orthonormal system to be considered will be that corresponding to unit weight function for integration with respect to $t$. On a segment of the curve not including or extending to the cusp, integration with respect to $t$ and integration with respect to $s$ are equivalent from the point of view of the application of Korous's theorem (see [7, pp. 352-353]).

Let $p_{0}(t), p_{1}(t), p_{2}(t), \cdots$ be the normalized Legendre polynomials in $t$ on the interval $a \leqq t \leqq b$, with the properties

$$
\int_{a}^{b} p_{m}(t) p_{n}(t) d t=0, \quad m \neq n \quad \int_{a}^{b}\left[p_{n}(t)\right]^{2} d t=1,
$$

and let $q_{0}(t), q_{2}(t), q_{3}(t), \ldots$ be the normalized orthogonal linear combinations of the powers of $t$ with omission of the first.

As a polynomial of the $n$th degree, $q_{n}(t)$ is linearly expressible in terms of $p_{0}, p_{1}, \cdots, p_{n}$ : 
with

$$
q_{n}(t)=\sum_{k=0}^{n} c_{n k} p_{k}(t)
$$

$$
c_{n k}=\int_{a}^{b} q_{n}(t) p_{k}(t) d t .
$$

As a member of the orthonormal system it is orthogonal to every polynomial of lower degree which satisfies the auxiliary condition. Such a polynomial in particular is $p_{k}(t)-t p_{k}^{\prime}(0)$; consequently

$$
\int_{a}^{b} q_{n}(t)\left[p_{k}(t)-t p_{k}^{\prime}(0)\right] d t=0, \quad c_{n k}=p_{k}^{\prime}(0) \int_{a}^{b} t q_{n}(t) d t
$$

for $k=0,1, \cdots, n-1$. (It is immaterial in the next formulas whether the fact that $c_{n 0}=0$ for $n>0$ is explicitly taken into account or not.)

Since

$$
\sum_{k=0}^{n} c_{n k}^{2}=\int_{a}^{b}\left[\sum_{k=0}^{n} c_{n k} p_{k}(t)\right]^{2} d t=\int_{a}^{b}\left[q_{n}(t)\right]^{2} d t=1
$$

it follows on the one hand that $\left|c_{n n}\right| \leqq 1$ (and, for subsequent reference, $\left|c_{n, n-1}\right| \leqq 1,\left|c_{n, n-2}\right| \leqq 1$ ) and on the other hand that

$$
\begin{gathered}
\sum_{k=0}^{n-1} c_{n k}^{2}=\left[\int_{a}^{b} t q_{n}(t) d t\right]^{2} \sum_{k=0}^{n-1}\left[p_{k}^{\prime}(0)\right]^{2} \leqq 1, \\
\left|\int_{a}^{b} t q_{n}(t) d t\right| \leqq\left\{\sum_{k=0}^{n-1}\left[p_{k}^{\prime}(0)\right]^{2}\right\}^{-1 / 2} .
\end{gathered}
$$

It is convenient in proceeding with the analysis to break up the expression for $q_{n}(t)$ into parts according to the formulas

$$
\begin{aligned}
q_{n}(t)= & c_{n n} p_{n}(t)+c_{n, n-1} p_{n-1}(t)+c_{n, n-2} p_{n-2}(t) \\
& +\int_{a}^{b} t q_{n}(t) d t \sum_{k=0}^{n-3} p_{k}^{\prime}(0) p_{k}(t), \\
\left|q_{n}(t)\right| \leqq & \left|c_{n n} p_{n}(t)\right|+\left|c_{n, n-1} p_{n-1}(t)\right|+\left|c_{n, n-2} p_{n-2}(t)\right| \\
& +\left|\sum_{k=0}^{n-3} p_{k}^{\prime}(0) p_{k}(t)\right|\left\{\sum_{k=0}^{n-1}\left[p_{k}^{\prime}(0)\right]^{2}\right\}^{-1 / 2} .
\end{aligned}
$$

By the Christoffel-Darboux identity,

$$
\sum_{k=0}^{n-3} p_{k}(u) p_{k}(t)=\frac{a_{n-3}}{a_{n-2}} \frac{p_{n-2}(u) p_{n-3}(t)-p_{n-3}(u) p_{n-2}(t)}{u-t},
$$

the symbol $a_{k}$ denoting generally the coefficient of $t^{k}$ in $p_{k}(t)$, taken by definition as positive. Hence 


$$
\begin{aligned}
\sum_{k=0}^{n-3} p_{k}^{\prime}(u) p_{k}(t)= & \frac{a_{n-3}}{a_{n-2}} \frac{p_{n-2}^{\prime}(u) p_{n-3}(t)-p_{n-3}^{\prime}(u) p_{n-2}(t)}{u-t} \\
& -\frac{a_{n-3}}{a_{n-2}} \frac{p_{n-2}(u) p_{n-3}(t)-p_{n-3}(u) p_{n-2}(t)}{(u-t)^{2}} \\
\left|\sum_{k=0}^{n-3} p_{k}^{\prime}(0) p_{k}(t)\right| \leqq & \frac{a_{n-3}}{a_{n-2}}\left|p_{n-2}^{\prime}(0)\right|\left|\frac{p_{n-3}(t)}{t}\right|+\frac{a_{n-3}}{a_{n-2}}\left|p_{n-3}^{\prime}(0)\right|\left|\frac{p_{n-2}(t)}{t}\right| \\
& +\frac{a_{n-3}}{a_{n-2}}\left|p_{n-2}(0)\right|\left|\frac{p_{n-3}(t)}{t^{2}}\right|+\frac{a_{n-3}}{a_{n-2}}\left|p_{n-3}(0)\right|\left|\frac{p_{n-2}(t)}{t^{2}}\right| .
\end{aligned}
$$

In the right-hand member of the last relation, $a_{n-3} / a_{n-2}$ has an upper bound independent of $n$, being not greater than the larger of the numbers $|a|,|b|$ (see for example [6, p. 195]); $\left|p_{n-2}(t)\right|$ and $\left|p_{n-3}(t)\right|$ are uniformly bounded throughout any closed interval interior to $(a, b)$; and the factors $1 / t$ and $1 / t^{2}$ of course are bounded except near $t=0$. Furthermore,

$$
\left|p_{n-2}^{\prime}(0)\right| \leqq\left\{\sum_{k=0}^{n-1}\left[p_{k}^{\prime}(0)\right]^{2}\right\}^{1 / 2}, \quad\left|p_{n-3}^{\prime}(0)\right| \leqq\left\{\sum_{k=0}^{n-1}\left[p_{k}^{\prime}(0)\right]^{2}\right\}^{1 / 2},
$$

the sum of non-negative terms inside the braces being at least as great as any single one of the terms; and similarly, for reference in the next paragraph, $\left|p_{n-1}^{\prime}(0)\right|$ and $\left|p_{n-4}^{\prime}(0)\right|$ have the same upper bound; since the question is that of boundedness as $n$ becomes infinite, there is no loss in supposing $n \geqq 4$, to avoid explanations connected with the superficial appearance of negative subscripts. It remains to compare the factors $p_{n-2}(0), p_{n-3}(0)$ as to order of magnitude with the expression in braces.

According to the recurrence relation connecting the $p$ 's,

$$
t p_{k}(t)=\alpha_{k, k+1} p_{k+1}(t)+\alpha_{k k} p_{k}(t)+\alpha_{k, k-1} p_{k-1}(t)
$$

with $\alpha_{k i}=\int_{a}^{b} t p_{k}(t) p_{j}(t) d t$. It is of course easy in the case of the Legendre polynomials to give the values of the $\alpha$ 's explicitly, but that is not necessary for the present purpose. By the application of Schwarz's inequality leading to the upper bound for $a_{n-3} / a_{n-2}$ which was used in the preceding paragraph, if $G$ is the larger of $|a|,|b|$,

$$
\left|\alpha_{k j}\right| \leqq G \int_{a}^{b}\left|p_{k}(t) p_{j}(t)\right| d t \leqq G\left\{\int_{a}^{b}\left[p_{k}(t)\right]^{2} d t \int_{a}^{b}\left[p_{j}(t)\right]^{2} d t\right\}^{1 / 2}=G .
$$

Hence on differentiation of the recurrence formula

$$
\begin{gathered}
p_{k}(t)+t p_{k}^{\prime}(t)=\alpha_{k, k+1} p_{k+1}^{\prime}(t)+\alpha_{k k} p_{k}^{\prime}(t)+\alpha_{k, k-1} p_{k-1}^{\prime}(t), \\
\left|p_{k}^{\prime}(0)\right| \leqq G\left\{\left|p_{k+1}^{\prime}(0)\right|+\left|p_{k}^{\prime}(0)\right|+\left|p_{k-1}^{\prime}(0)\right|\right\} \leqq 3 G\left\{\sum_{k=0}^{n-1}\left[p_{k}^{\prime}(0)\right]^{2}\right\}^{1 / 2}
\end{gathered}
$$

for $k=n-2, n-3$, and in general when $k+1 \leqq n-1$. 
From the inequalities of the last two paragraphs it follows that

$$
\left|\sum_{k=0}^{n-3} p_{k}^{\prime}(0) p_{k}(t)\right|\left\{\sum_{k=0}^{n-1}\left[p_{k}^{\prime}(0)\right]^{2}\right\}^{-1 / 2}
$$

is uniformly bounded throughout any closed interval interior to $(a, b)$, except in the neighborhood of the point $t=0$ if this point is inside the interval; and hence that $q_{n}(t)$ is similarly bounded.

The results can be interpreted immediately with respect to the orthonormal polynomials in $x$ and $y$ which reduce to the respective $q$ 's for points $(x, y)$ on the curve.

5. The curve $y^{3}=x^{4}$, symmetric arc. The reasoning of the last section with regard to the boundedness of orthonormal polynomials lacking the first power of the independent variable retains its validity if a weight function satisfying the usual conditions is admitted in the definition of orthogonality. Thus generalized, it supplies the information needed for dealing with orthogonal polynomials on a symmetric arc of the curve $y^{3}=x^{4}$.

Since any monomial having $x^{4}$ as a factor can be replaced on the curve by one of lower degree, the orthogonal system can be constructed from the monomials

$$
1, x, y, x^{2}, x y, y^{2}, x^{3}, x^{2} y, x y^{2}, y^{3}, x^{3} y, x^{2} y^{2}, x y^{3}, y^{4}, \cdots .
$$

In terms of the parametric representation $x=t^{3}, y=t^{4}$, these become $1, t^{3}, t^{4}, t^{6}$, and the succeeding integral powers of $t$ in natural order without further omission. On an interval $-a \leqq t \leqq a$ it suffices to consider separately even polynomials formed from the powers $1, t^{4}, t^{6}, t^{8}, \cdots$ and odd polynomials formed from the powers $t^{3}, t^{7}, t^{9}, t^{11}, \cdots$. Let the corresponding members of the orthonormal system be denoted respectively by $r_{0}(t), r_{4}(t), r_{6}(t), \cdots$ and $r_{3}(t), r_{7}(t), r_{9}(t), \cdots$.

Let $u=t^{2}, c=a^{2}$, and let $r_{2 i}(t)=q_{i}(u)$. Then, whether $i$ and $j$ are the same. or different,

$$
\int_{-a}^{a} r_{2 i}(t) r_{2 j}(t) d t=2 \int_{0}^{a} r_{2 i}(t) r_{2 j}(t) d t=\int_{0}^{c} u^{-1 / 2} q_{i}(u) q_{j}(u) d u .
$$

The $r$ 's are orthonormal for unit weight on the interval $(-a, a)$ if the $q$ 's are orthonormal with weight $u^{-1 / 2}$ for $0 \leqq u \leqq c$. The $q$ 's then constitute the orthonormal system in terms of the powers of $u$ with omission of the first, for the weight function specified, and are uniformly bounded except near the ends of the interval, by the observation in the opening sentence of this section, since the corresponding Jacobi polynomials formed from the complete sequence of non-negative powers of $u$ are thus bounded (in fact without exclusion of the left-hand end of the interval). Hence the $r$ 's are uniformly bounded when $t$ remains at a distance from the points $-a, 0, a$. 
With a change of notation as regards the $q$ 's let $r_{2 i+3}(t)=t^{3} q_{i}(u)$. For any values of $i$ and $j$, alike or different, from the sequence $0,2,3,4, \cdots$,

$$
\int_{-a}^{a} r_{2 i+3}(t) r_{2 j+3}(t)=2 \int_{0}^{a} r_{2 i+3}(t) r_{2 j+3}(t) d t=\int_{0}^{c} u^{5 / 2} q_{i}(u) q_{j}(u) d u .
$$

The $q$ 's, orthonormal combinations of $1, u^{2}, u^{3}, \cdots$ with weight $u^{5 / 2}$, are uniformly bounded except near the ends of the interval $(0, c)$ for $u$, and the $r$ 's are uniformly bounded except near the ends and middle point of the interval $(-a, a)$ for $t$.

The orthonormal system on the curve, consisting of the even and odd $r$ 's together as expressed in terms of $x$ and $y$, is uniformly bounded except near the ends and middle point of the arc.

6. The curve $y^{2}=x^{5}$, arbitrary arc. Omission of two powers of the independent variable. By the next to the last paragraph of $\$ 3$, together with the earlier observations about permutation of the functions in a sequence, the problem of boundedness of the orthonormal polynomials in $x$ and $y$ on an arbitrary arc of the curve $y^{2}=x^{5}$ is equivalent to that of the orthonormal polynomials formed from the powers of $t$ with omission of the first and third, on an arbitrary interval $a \leqq t \leqq b$.

Let $q_{0}(t), q_{2}(t), q_{3}(t), \cdots$ denote the orthonormal polynomials of $\S 4$, formed from all the non-negative integral powers of $t$ except the first. For convenience and uniformity in writing formulas of summation let $q_{1}(t) \equiv 0$. Let $r_{0}(t), r_{2}(t), r_{4}(t), r_{5}(t), \cdots$ be the orthonormal polynomials of the new problem. The exclusion of $t$ and $t^{3}$ in their construction is equivalent to subjecting them to the pair of auxiliary conditions

$$
r_{n}^{\prime}(0)=r_{n}^{\prime \prime \prime}(0)=0 .
$$

Since every polynomial which satisfies the single auxiliary condition characterizing the $q$ 's, namely the vanishing of the first derivative for $t=0$, can be expressed linearly in terms of the $q$ 's, $r_{n}(t)$ in particular has a representation of the form

with

$$
r_{n}(t)=\sum_{k=0}^{n} c_{n k} q_{k}(t)
$$

$$
c_{n k}=\int_{a}^{b} r_{n}(t) q_{k}(t) d t
$$

(entirely distinct, of course, from the $c_{n k}$ of $\S 4$ ). For literal accuracy in certain subsequent statements it may be understood in particular that $c_{n 1}=0$.

By virtue of its own characteristic properties, $r_{n}(t)$ is orthogonal to every polynomial of lower degree whose first and third derivatives vanish for $t=0$. Such a polynomial is $q_{k}(t)-t^{3} q_{k}^{\prime \prime \prime}(0) / 6$. Consequently, for $k<n$, 


$$
\int_{a}^{b} r_{n}(t)\left[q_{k}(t)-\frac{1}{6} t^{3} q_{k}^{\prime \prime \prime}(0)\right] d t=0, \quad c_{n k}=\frac{1}{6} q_{k}^{\prime \prime \prime}(0) \int_{a}^{b} t^{3} r_{n}(t) d t .
$$

Since

$$
\sum_{k=0}^{n} c_{n k}^{2}=\int_{a}^{b}\left[\sum_{k=0}^{n} c_{n k} q_{k}(t)\right]^{2} d t=\int_{a}^{b}\left[r_{n}(t)\right]^{2} d t=1,
$$

each of the $c$ 's individually has 1 as an upper bound for its magnitude. Furthermore,

$$
\begin{gathered}
\sum_{k=0}^{n-1} c_{n k}^{2}=\frac{1}{36}\left[\int_{a}^{b} t^{3} r_{n}(t) d t\right]^{2} \sum_{k=0}^{n-1}\left[q_{k}^{\prime \prime \prime}(0)\right]^{2} \leqq 1, \\
\left|\int_{a}^{b} t^{3} r_{n}(t) d t\right| \leqq 6\left\{\sum_{k=0}^{n-1}\left[q_{k}^{\prime \prime \prime}(0)\right]^{2}\right\}^{-1 / 2} .
\end{gathered}
$$

(If the question is raised whether the sum in braces could vanish, a sufficient answer is that in that case $c_{n k}$ would vanish for $k=0, \cdots, n-1$, and the desired conclusion with regard to the boundedness of $r_{n}(t)$ would follow with trivial facility.)

Let the expression for $r_{n}(t)$ in terms of the $q$ 's be separated into parts according to the formula

$$
r_{n}(t)=\sum_{k=n-4}^{n} c_{n k} q_{k}(t)+\frac{1}{6} \int_{a}^{b} t^{3} r_{n}(t) d t \sum_{k=0}^{n-5} q_{k}^{\prime \prime \prime}(0) q_{k}(t) ;
$$

to avoid negative subscripts in a literal reading of the formulas it will be convenient ultimately to assume $n \geqq 8$, which is legitimate inasmuch as the question at issue concerns behavior as $n$ becomes infinite. An upper bound for $\left|r_{n}(t)\right|$ is to be obtained from the relation

$$
\left|r_{n}(t)\right| \leqq \sum_{k=n-4}^{n}\left|c_{n k} q_{k}(t)\right|+\left|\sum_{k=0}^{n-5} q_{k}^{\prime \prime \prime}(0) q_{k}(t)\right|\left\{\sum_{k=0}^{n-1}\left[q_{k}^{\prime \prime \prime}(0)\right]^{2}\right\}^{-1 / 2}
$$

It is to be noted here that the number of terms in the first summation on the right does not increase with $n$, and in consequence of the boundedness of the $c$ 's, pointed out in the preceding paragraph, and the boundedness of the $q$ 's as recognized in $\$ 4$ the first sum itself has an upper bound independent of $n$ throughout any closed interval interior to $(a, b)$ and excluding the point $t=0$, if this point is in the interval.

While the setting up of a relation of recurrence for the $q$ 's is complicated by the fact that $t q_{k}(t)$ does not in general satisfy the auxiliary condition to which the $q$ 's are subject, namely that of having a vanishing derivative for $t=0$, this condition is satisfied by $t^{2} q_{k}(t)$, and there is a relation (see $[5, \mathrm{pp} .74$ 75]) of the form 


$$
t^{2} q_{k}(t)=\sum_{j=k-2}^{k+2} \alpha_{k j} q_{j}(t)
$$

with

$$
\alpha_{k j}=\int_{a}^{b} t^{2} q_{k}(t) q_{j}(t) d t
$$

If $G$ is the larger of the quantities $|a|,|b|$,

$$
\left|\alpha_{k j}\right| \leqq G^{2} \int_{a}^{b}\left|q_{k}(t) q_{j}(t)\right| d t \leqq G^{2}\left\{\int_{a}^{b}\left[q_{k}(t)\right]^{2} d t \int_{a}^{b}\left[q_{j}(t)\right]^{2} d t\right\}^{1 / 2}=G^{2} .
$$

From the recurrence relation is derived a Christoffel-Darboux identity

$$
\sum_{k=0}^{n} q_{k}(u) q_{k}(t)=\sum_{n}^{\prime} \alpha_{i j} \frac{q_{i}(u) q_{j}(t)-q_{j}(u) q_{i}(t)}{u^{2}-t^{2}}
$$

in which the summation $\sum_{n}^{\prime}$ extends over the three pairs of values $(n+2, n)$, $(n+1, n)$ and $(n+1, n-1)$ for $i$ and $j$.

For use in (5), $n$ as upper limit of summation in the left-hand member of the last identity is to be replaced by $n-5$, and the summation on the right is to cover the pairs of indices $(n-3, n-5),(n-4, n-5),(n-4, n-6)$. By repeated differentiation with respect to $u$, we get

$$
\begin{aligned}
\sum_{k=0}^{n-5} q_{k}^{\prime \prime \prime}(u) q_{k}(t)= & \sum_{n=5}^{\prime} \alpha_{i j} \frac{q_{i}^{\prime \prime \prime}(u) q_{j}(t)-q_{j}^{\prime \prime \prime}(u) q_{i}(t)}{u^{2}-t^{2}} \\
& -6 \sum_{n=5}^{\prime} \alpha_{i j} \frac{u}{\left(u^{2}-t^{2}\right)^{2}}\left[q_{i}^{\prime \prime}(u) q_{j}(t)-q_{j}^{\prime \prime}(u) q_{i}(t)\right] \\
& -6 \sum_{n=5}^{\prime} \alpha_{i j}\left[\frac{1}{\left(u^{2}-t^{2}\right)^{2}}-\frac{4 u^{2}}{\left(u^{2}-t^{2}\right)^{3}}\right]\left[q_{i}^{\prime}(u) q_{j}(t)-q_{j}^{\prime}(u) q_{i}(t)\right] \\
& +\sum_{n=5}^{\prime} \alpha_{i j}\left[q_{i}(u) q_{j}(t)-q_{j}(u) q_{i}(t)\right] \frac{\partial^{3}}{\partial u^{3}} \frac{1}{u^{2}-t^{2}} .
\end{aligned}
$$

The first and third derivatives of $1 /\left(u^{2}-t^{2}\right)$ as an even function of $u$ are odd functions of $u$, and vanish on substitution of the value $u=0$. Hence

$$
\begin{aligned}
\sum_{k=0}^{n-5} q_{k}^{\prime \prime \prime}(0) q_{k}(t)= & -\sum_{n=5}^{\prime} \alpha_{i j}\left[q_{i}^{\prime \prime \prime}(0) q_{j}(t)-q_{j}^{\prime \prime \prime}(0) q_{i}(t)\right] / t^{2} \\
& -6 \sum_{n=5}^{\prime} \alpha_{i j}\left[q_{i}^{\prime}(0) q_{j}(t)-q_{j}^{\prime}(0) q_{i}(t)\right] / t^{4} .
\end{aligned}
$$

As $n$ increases, the number of terms under each sign of summation on the right remains bounded; the values of $\left|\alpha_{i j}\right|$ have the fixed upper bound $G^{2}$; 
if $t$ is restricted to a closed interval contained in $(a, b)$ and excluding the values $a, b$, and $0,\left|q_{i}(t)\right|$ and $\left|q_{j}(t)\right|$ are bounded, according to $\S 4$, and $1 / t^{2}$ and $1 / t^{4}$ are bounded; and since $i$ and $j$ are restricted to the values $n-3, n-4$, $n-5, n-6$,

$$
\left|q_{i}^{\prime \prime \prime}(0)\right| \leqq\left\{\sum_{k=0}^{n-1}\left[q_{k}^{\prime \prime \prime}(0)\right]^{2}\right\}^{1 / 2}, \quad\left|q_{j}^{\prime \prime \prime}(0)\right| \leqq\left\{\sum_{k=0}^{n-1}\left[q_{k}^{\prime \prime \prime}(0)\right]^{2}\right\}^{1 / 2} .
$$

Finally, by three differentiations of (6), with replacement of the subscript $k$ by $i$,

$t^{2} q_{i}^{\prime \prime \prime}(t)+6 t q_{i}^{\prime \prime}(t)+6 q_{i}^{\prime}(t)$

$$
=\alpha_{i, i-2} q_{i-2}^{\prime \prime \prime}(t)+\alpha_{i, i-1} q_{i-1}^{\prime \prime \prime}(t)+\cdots+\alpha_{i, i+2} q_{i+2}^{\prime \prime \prime}(t),
$$

and as $i+2 \leqq n-1$,

$$
6\left|q_{i}^{\prime}(0)\right| \leqq 5 G^{2}\left\{\sum_{k=0}^{n-1}\left[q_{k}^{\prime \prime \prime}(0)\right]^{2}\right\}^{1 / 2},
$$

while the same inequality holds if the index $i$ is replaced by $j$.

Consequently, in the right-hand member of (5)

$$
\left|\sum_{k=0}^{n-5} q_{k}^{\prime \prime \prime}(0) q_{k}(t)\right|\left\{\sum_{k=0}^{n-1}\left[q_{k}^{\prime \prime \prime}(0)\right]^{2}\right\}^{-1 / 2}
$$

remains uniformly bounded as $n$ increases if $t$ is restricted to a closed interior subinterval of $(a, b)$ excluding the point $t=0$, and $\left|r_{n}(t)\right|$ is similarly bounded.

It is clear that the above method can be employed more generally, and also that its formulation in general terms would be awkward and laborious.

7. Curves in three dimensions. Although the above inquiry was undertaken primarily with reference to plane curves, it turns out that the auxiliary developments are equally applicable to the study of orthonormal polynomials on certain skew curves in three-dimensional space [3] (or in space of a larger number of dimensions, but that extension will not be further discussed here). The curves will be taken as defined initially in terms of parametric representation, and interpretation in connection with the intersection of surfaces will be incidental.

On the curve

$$
x=t, \quad y=t^{2}, \quad z=t^{3},
$$

the coordinates satisfy the relations $y=x^{2}, z=x y$, and the curve is the intersection of the corresponding quadric surfaces. Any monomial containing $x^{2}$ or $x y$ as a factor can be replaced on the curve by one of lower degree. The equation $y^{2}=x z$, representing another quadric surface which passes through the curve, makes it possible to replace $y^{2}$, not by an expression of lower degree to be sure, but by one of lower rank, in accordance with a convention 
which has been used in connection with the study of orthogonal systems on algebraic curves in space; see [3, pp. 443-444, 447-449]. In the application of the Schmidt process consideration may be limited to terms of the first degree at most in the pair of variables $x, y$. The monomials

$$
1, x, y, z, x z, y z, z^{2}, x z^{2}, y z^{2}, z^{3}, \cdots
$$

reduce in terms of the parametric representation to the successive powers of $t$, without omission and in natural order. They are certainly linearly independent on the curve, since a relation of linear dependence connecting any number of them would express the identical vanishing of a polynomial in $t$ with coefficients not all zero. The orthonormal polynomials in $x, y, z$ on any arc of the curve are the normalized Legendre polynomials on the corresponding interval of values of $t$, and are uniformly bounded accordingly except near the ends of the arc.

On the curve

$$
x=t, \quad y=t^{2}, \quad z=t^{4},
$$

the relations $y=x^{2}, z=y^{2}$, expressing the fact that this curve again is an intersection of two quadrics, serve to eliminate from consideration in the setting up of the orthogonal system all monomials which are of degree higher than the first in $x$ or $y$ separately. The remaining monomials

$$
1 ; x, y, z ; x y, x z, y z, z^{2} ; x y z, x z^{2}, y z^{2}, z^{3} ; \cdots,
$$

each of which after the first eight is obtained by multiplying the fourth preceding by $z$, reduce in terms of the parametric representation to

$$
1 ; t, t^{2}, t^{4} ; t^{3}, t^{5}, t^{6}, t^{8} ; t^{7}, t^{9}, t^{10}, t^{12}, \cdots,
$$

a permutation of the non-negative powers of $t$ in which no power is displaced more than one step from its natural position. Since the powers included in the sequence are all different, no finite number of them can be connected by any relation of linear dependence. The orthonormal polynomials constructed for any finite interval from the members of the sequence in the order indicated, whether expressed in terms of $t$ or in terms of $x, y, z$, are uniformly bounded except near the ends of the range of integration.

In the case of the curve

$$
x=t, \quad y=t^{3}, \quad z=t^{4},
$$

the equations $y=x^{3}, z=x y$, identifying the curve as intersection of a cylindrical surface of the third degree with a hyperbolic paraboloid, together with the supplementary relations $x^{2} z=y^{2}, y^{3}=x z^{2}$, reduce the list of monomials requiring independent consideration to

$$
1 ; x, y, z ; x^{2}, x z, y^{2}, y z, z^{2} ; x z^{2}, y^{2} z, y z^{2}, z^{3} ; \cdots,
$$

each of which after the first nine is obtained by multiplying the fourth preced- 
ing one by $z$. As these become, in terms of the parameter, $1, t, t^{3}, t^{4}, t^{2}, t^{5}$, followed by the remaining powers of $t$ in natural order, it is clear even without reference to the details of the process by which they were selected that they form a complete basis for the construction of the orthonormal system. The polynomials of the system from the one of the fifth degree on are the normalized Legendre polynomials of corresponding degree for the interval considered, and are bounded accordingly throughout any closed interior interval. (It may be noted that the list (7) includes five monomials of the second degree, and not more than four of any other degree.)

Two more illustrations may serve to suggest the further scope of this particular procedure. The equations

$$
x=t, \quad y=t^{2}, \quad z=t^{5}
$$

describe the intersection of the surfaces $y=x^{2}, z=x y^{2}$. Fundamental monomials are

$$
1 ; x, y, z ; x y, x z, y^{2}, y z, z^{2} ;
$$

followed by the products of the last five by successive powers of $z$; or in terms of the parameter

$$
1 ; t, t^{2}, t^{5} ; t^{3}, t^{6}, t^{4}, t^{7}, t^{10}
$$

with the products of the last five by powers of $t^{5}$. In the latter sequence the inclusion of $t^{6}, t^{7}, t^{8}, t^{9}, t^{10}$, together with the product of each by an arbitrary positive integral power of $t^{5}$, and the presence of the terms $1, t, t^{2}, t^{3}, t^{4}, t^{5}$ as listed individually, without repetition, guarantee the completeness of the list and the linear independence of its members. Furthermore, no power is displaced more than two steps from its normal position. For the curve

$$
x=t, \quad y=t^{2}, \quad z=t^{6},
$$

the intersection of the surfaces $y=x^{2}, z=y^{3}$, fundamental monomials are

$$
1 ; x, y, z ; x y, x z, y^{2}, y z, z^{2} ; x y^{2}, x y z, x z^{2}, y^{2} z, y z^{2}, z^{3} ;
$$

with the products of the last six by powers of $z$; or

$$
1 ; t, t^{2}, t^{6} ; t^{3}, t^{7}, t^{4}, t^{8}, t^{12} ; t^{5}, t^{9}, t^{13}, t^{10}, t^{14}, t^{18}
$$

and multiples of the last six by powers of $t^{6}$. All non-negative powers of $t$ are present, and none is more than four steps out of place, the discrepancies manifested in the case of the powers $t^{5}, t^{9}, \cdots, t^{18}$ being repeated in each succeeding group of six. In each case the method of $\$ 2$ suffices to guarantee the boundedness of the orthonormal polynomials except near the ends of the interval or arc.

Let attention be turned now to the curve

$$
x=t^{2}, \quad y=t^{3}, \quad z=t^{4},
$$


the intersection of the quadrics $z=x^{2}, y^{2}=x z$, parabolic cylinder and cone, which are tangent to each other at the origin in such a way that their intersection has a cusp there. It is clear that no monomial in $x, y$, and $z$ can reduce to the first power of $t$, while

$$
1 ; x, y, z ; x y, x z, y z, z^{2} ;
$$

and the multiples of the last four by successive powers of $z$ are equal to $1, t^{2}, t^{3}, t^{4}$, and the succeeding powers of $t$ in natural order. The orthonormal polynomials are those formed from the powers of $t$ with omission of the first, and their properties of boundedness can be recognized by the methods of $\S \S 3$ and 4.

On the curve

$$
x=t^{2}, \quad \cdot y=t^{3}, \quad z=t^{5},
$$

intersection of the surfaces $z=x y$ and $y^{2}=x^{3}$, no monomial in terms of the coordinates can be equal to the first power of $t$, but the monomials

$$
1 ; x, y, z ; x^{2}, x z, y^{2}, y z, z^{2} ;
$$

and the products of the last five by successive powers of $z$ are equal respectively to

$$
1 ; t^{2}, t^{3}, t^{5} ; t^{4}, t^{7}, t^{6}, t^{8}, t^{10}
$$

and multiples of the last five by powers of $t^{5}$. Again all powers of $t$ are represented except the first, with none displaced more than one step from the position which it would occupy if the exponents were arranged in natural order, so that the previous analysis is applicable again. Results are similar in the case of the curve

$$
x=t^{2}, \quad y=t^{3}, \quad z=t^{6}:
$$

$z=x^{3}=y^{2}$. Independent monomials are

$$
1 ; x, y, z ; x^{2}, x y, x z, y z, z^{2} ; x^{2} y, x^{2} z, x y z, x z^{2}, y z^{2}, z^{3} ;
$$

and multiples of the last six by powers of $z$; these reduce to the powers of $t$ with the first omitted, and with none moved more than three steps from its natural position in order of magnitude of the exponents.

For an application of the results of $\S 6$ reference may be made to the curve

$$
x=t^{2}, \quad y=t^{4}, \quad z=t^{5},
$$

forming the intersection of the surfaces $y=x^{2}, z^{2}=x y^{2}$. Independent monomials are

$$
1 ; x, y, z ; x y, x z, y^{2}, y z, z^{2} ;
$$

and multiples of the last five by powers of $z$, reducing in terms of the parameter to $1, t^{2}, t^{4}, t^{5}, t^{6}$, and all the succeeding powers of $t$ in natural order. As a final illustration, the curve 


$$
x=t^{2}, \quad y=t^{5}, \quad z=t^{6},
$$

or in terms of the coordinates $z=x^{3}, y^{2}=x^{2} z$, has as a fundamental system of monomials for the construction of its orthogonal system

$$
\begin{gathered}
1 ; x, y, z ; x^{2}, x y, x z, y^{2}, y z, z^{2} ; \\
x^{2} y, x y z, x z^{2}, y^{3}, y^{2} z, y z^{2}, z^{3} ; x y z^{2}, x z^{3}, y^{3} z, y^{2} z^{2}, y z^{3}, z^{4} ;
\end{gathered}
$$

and multiples of the last six by powers of $z$, a list including seven terms of the third degree and six of each other degree above the first. In terms of the parameter the monomials are

$$
1 ; t^{2}, t^{5}, t^{6} ; t^{4}, t^{7}, t^{8}, t^{10}, t^{11}, t^{12} ; t^{9}, t^{13}, t^{14},
$$

followed by the higher powers of $t$ in natural order. All powers are included except the first and third, and the conclusions of $\$ 6$ are applicable once more.

\section{REFERENCES}

1. G. E. Albert and L. H. Miller, Equiconvergence theorems for orthonormal polynomials, Bull. Amer. Math. Soc. vol. 50 (1944) pp. 358-367.

2. D. Jackson, Orthogonal polynomials on a plane curve, Duke Math. J. vol. 3 (1937) pp. 228-236. 454.

3. - Orthogonal polynomials in three variables, Duke Math. J. vol. 4 (1938) pp. 441-

4. - A new class of orthogonal polynomials, Amer. Math. Monthly vol. 46 (1939) pp. $493-497$.

5. - Orthogonal polynomials with auxiliary conditions, Trans. Amer. Math. Soc. vol. 48 (1940) pp. 72-81.

6. - Fourier series and orthogonal polynomials, Carus Mathematical Monographs, No. 6, 1941.

7. Boundedness of orthonormal polynomials on loci of the second degree, Duke Math. J. vol. 11 (1944) pp. 351-365.

8. F. Koehler, Systems of orthogonal polynomials on certain algebraic curves, Bull. Amer. Math. Soc. vol. 46 (1940) pp. 345-351. 1939.

9. G. Szegö, Orthogonal polynomials, Amer. Math. Soc. Colloquium Publications, vol. 23,

UNIVERSITY OF MinNESOTA, Minneapolis, MinN. 